\title{
Study on pricing mechanism of pumped hydro energy storage (PHES) under China's electricity tariff reform
}

\author{
Fuqiang Zhang*, Zhicheng Xu, Bingqi Jiao and Junshu Feng \\ State Grid Energy Reasearch Institute CO., LTD., Beijing, 102209, China
}

\begin{abstract}
This paper presents a pricing mechanism for pumped hydro energy storage (PHES) to promote its healthy development. The proposed pricing mechnism includes PHES pricing mechanism and cost sharing mechanism. Regarding the PHES pricing mechanism, the existed two-part tariff is still recommended to implement at the current and future stages. Regarding the cost sharing mechanism, it is suggested that the electricity capacity fee and pumping-loss fee should be all included in the allowable transmission and distribution costs of the regional power grids, which can be further transmitted to the provincial power grids. At the same time, we also suggest to exploring other funding sources to relieve the pressure of PHES cost sharing totally through the electricity sales price.
\end{abstract}

\section{Introduction}

In China, the capacity fees and pumping losses of the PHES were included in the operating costs of the local provincial power grid (or regional power grid) according to the policy document Notice on Improving Price Formation Mechanism of Pumped Hydro Energy Storage (No.1763), which was released in 2014 by the Development and Reform Commission of China (NDRC) [1]. The settlement of electricity charge between the PHES and power grid enterprises will be conducted in accordance with the price standards approved by the government price authorities. In December 2016, the National Development and Reform Commission of China promulgated the Measures for Electricity Tariff Determination for Transmission and Distribution of Provincial Power Grid (Trial) with the promotion of transmission and distribution tariff reform [2]. It clearly states that the relevant costs of a PHES were not included in the allowable income of the power grid company, but how to share the cost was not stipulated in detail. Consequently, the new-built PHESs are now facing the challenge that the annual operating costs (AOC) cannot be properly settled, which results in affecting the healthy development of the PHES industry. For example, at present, $25 \%$ of the capacity fees of $\mathrm{Hei}$ Mifeng PHES cannot be settled normally, which results in a loss of 445 million Yuan accumulately. For other PHESs which are built or put into operation recently in Zhejiang and Jiangxi provinces, their capacity fees still have not been normally settled.

In order to develop a suitable pricing mechanism and electricity tariff revenue mechanism under the electricity market environment, this paper tries to put forward a reasonable scheme for the PHES. We first analyze and conclude the present price formation and tariff revenue mechanism for PHES. Secondly, an in-depth analysis of digging China's existing problems for the current electricity system reform has been conducted. Next, the feasibility of participation of various ancillary services of PHES into electricity market system has been analyzed. Then, an electricity tariff scheme for different stages of the electricity market is proposed for PHES under the electricity market. The proposed pricing mechanism will provide policy reference and detailed implementation measures for the healthy development of PHES in the electricity market environment.

\section{International PHES revenue mechanisms in different market phases}

\subsection{Existing international markets and their revenue mechanisms}

According to literature review and research, about $85 \%$ of the $150 \mathrm{GW}$ PHES in the world adopt the grid unified management mode or leasing mode, such as France, Japan and several states in the United States. In this way, the PHES is either built and operated by the power grid or built by a third party and leased out by the power grid and the related costs are incorporated into the unified accounting of the power grid and are shared through the sales price $[8,9]$.

About only $15 \%$ of the world's PHESs earn revenue by participating in the electricity market competition, typically such as RTO/ISO in the United Kingdom and the United States. PHESs are mostly funded by independent power plants (IPPs) or distribution (sales) utility companies that generate revenue by participating in the energy markets and ancillary service markets. However, the income received in the market can only

\footnotetext{
* Corresponding author: zhangfuqiang@sgeri.sgcc.com.cn
} 
cover $20 \%$ to $30 \%$ of its costs. It also needs to negotiate with the trading agencies to sign a medium and longterm contract for sale ancillary services to obtain a stable income, so as to guarantee the investment return. For the PHESs of Britain's First Hydroelectric Company, their annually fixed income parts of $70 \%$ to $80 \%$ are compensated by the British National Grid, and the surcharge (uplift) approach to user guidance; through participation in the energy market arbitrage variable obtained only $20 \%$ to $30 \%$, this part also covers the pumping station hair loss.

The main reasons why the global PHES is not involved in the competition in the electricity market are as follows: 1) Difficult to determine the benefits: The ancillary services such as emergency backup and black start provided by the PHES have a great effect on the safe and stable operation of the power system. However, the beneficiaries are extensive and difficult to quantify and characterize, so it is not easy to determine the compensation standard. 2) Market risk: In electricity market competition, PHES will face the risk brought by the volatility of electricity price, while investors generally prefer to invest in projects with a lower risk of investment. In the recent 10 years, with the rapid development of renewable energy such as wind and solar, the demand for storage power plant such as PHES has greatly increased. However, a majority of countries in the world face the problem of low enthusiasm for investment in PHES due to market risks. 3) Historical inertia: The vast majority of owners of PHESs built in the last century are vertically monopolized electricity companies, and still use the existing revenue mechanism that integrates PHES costs into the electricity sales price.

\subsection{PHES revenue mechanism in China and existing barriers to PHES development}

At present, the price of the existing PHESs in the operating area of the State Grid Corporation of China (SGCC) mainly consists of the two-part tariff and the single-capacity tariff. The relevant charges of the PHES have basically been covered by the sales price. As of the end of 2016, two of the 20 PHESs that belong to SGCC have put into operation mainly implemented two power price modes: one is the two-part tariff scheme, which consists of capacity-tariff and energy-tariff. The PHESs implement this kind of tariff including Zhejiang Tianhuangping, Liaoning Pushihe, Fujian Xianyou, Beijing Ming Tombs, Henan Huilong, Zhejiang Xianju, and Jiangxi Hongping PHESs. The capacity fee in the two-part tariff is approved by the NDRC of China and mainly reflects the ancillary service value of the PHES that provides power system reserve, regulation, and black start. The tariff will be settled between the provincial power grid company and the PHES, and the capacity fee is included in the local provincial level (or regional grid) operating expenses. The energy fee is mainly designed to make up for the variable costs such as PHES pumping and generation losses. In China, the pumping electricity for PHES is the $75 \%$ of local coalfired units benchmark electricity price (considering desulfurization, denitrification, dust and other environmental protection electricity prices, the same below). Other PHES plants adopt the single capacitytariff scheme at present $\mathrm{n}$ China, including Shandong Taishan, Zhejiang Tongbai, Jiangsu Yixing, Anhui Langya Mountain, Hebei Zhang Hewan, Henan Baoquan, Shanxi Xilong Chi, Hunan Hemifeng, Hebei Panjiakou, Jilin Baishan, Hubei Bailian River, Anhui Xiangshui, and Anhui Xiang Hongdian [10].

With the progress of power market reform, there are three main challenges and barriers to the healthy development of PHES, which are listed as follows:

(1) There is no reasonable pricing mechanism to ensure the healthy development of PHESs

The measures pointed out in [2] for "provincial power transmission and distribution pricing determination method " indicated that the PHES shall not be included in the effective asset of power systems. However, there is no clear regulation of what channel to pass through the costs of PHESs, so the newly put into operation and the unregulated PHESs are faced with the difficulty of the normal settlement of the electricity price. Therefore, it is necessary to study the price dredging mechanism of PHES which is suitable for the present stage.

(2) Few ancillary services can participate in market competition

Taking China's currently running ancillary services market as an example, such as Northeast Power Market, only two ancillary services (peak shaving and black-start) can participate in the market. There are too few ancillary services that can participate in the market competition, and the general ancillary services such as AGC and reserve service do not involve in present power market in China. Secondly, there is no discrepancy between the requirements of the adjustment rate of peak regulation service in the ancillary service market in Northeast China, and the advantages of the fast adjustment of the power plant can not be reflected. Thirdly, for the excess pumping power and black-start service provided by PHES, the administrative pricing method is used to give a small amount of compensation, which does not reflect the true market value.

(3) The sharing mechanism of "who benefits, who pay" has poor operability

According to the requirements of No. 9 issued by the Central Government of China, the principle of "who benefits, who pay" requires a sharing mechanism for each participated user should be established to offer and share the services. However, the implementation of the above mechanism has not yet been implemented in practice timely during the period of the reform of transmission and distribution pricing mechanism. There is a vacancy period of policy convergence, resulting in the situation that the newly-started PHESs cannot be settled normally. At the same time, the operational characteristics of PHES also determine that this mechanism is difficult to implement in the future ancillary service market. On the one hand, the benefits provided by PHES such as frequency regulation, phase modulation, accidental backup and black start are difficult to be accurately measured and calculated. On 
the other hand, the ancillary services for PHES have a variety of beneficiaries, including various types of thermal power plants, nuclear power, and renewable energy, as well as power grids and load consumers. In fact, the ancillary services offered by PHESs serve the entire power system and are difficult to accurately target specific beneficiaries. The wide range of beneficiaries led to the lack of maneuverability.

\section{The revenue scale calculation in different market phases}

In order to estimate whether the PHES is suitable for participating in the electricity market competition, we conduct a revenue scale calculation for a specific PHES if it is in a free electricity market in this part. In this way, we can prove that whether the main part of the investment and operation cost can be covered or not when it participates in the market competition.

\subsection{Market-oriented transitional phase}

In the transition period of the electricity market, the ancillary service market has not yet been fully established, so it is suitable for using fixed compensation mode to obtain ancillary services. According to the successful establishment of electric energy spot market or not, the electricity price and revenue mechanism in the transitional period can be divided into two situations: 1) only the medium and long-term electricity energy market; 2) the medium- and long-term electricity energy markets exist at the same time.

(1) Only the medium and long-term electric energy market

Due to the limited reservoir capacity of PHES, the plant cannot provide a continuous and stable power output. Therefore, it can only be profitable in the spot market of electric energy by short-term (intra-day and inter-period) price fluctuations, and cannot participate in medium- and long-term electric energy market.

(2) Both medium- and long-term electricity energy markets exist

If the spot market for electricity energy is established, the PHES can participate in the competition in the electricity energy market and obtain some benefits through providing peak shaving service. The ratio of the revenue scale from electricity energy market to the capacity fee is estimated below to provide a reference for the government price makers to make a reasonable pricing mechanism. In order to calculate the portion of benefits revenue from the electricity energy market, first of all, we need to determine the peak-valley electricity price ratio. Reference to the domestic and international electricity market operation experience shows that the market peak spot electricity price ratio is only about 1.5 to 2 times [11-13]. See Table 1 for details. It is noted that, when PHES participates in the competition of electricity energy market, it will reduce the peak-valley ration of origin power system. The main reason for this phenomenon is that PHES will ease the shortage of supply and demand at the peak of load and the oversupply situation at the time of low load as the PHES has the function of peak shaving. In general, the peakvalley electricity price difference can be reduced, and the reduction range can reach $10 \%$ to $30 \%$. Based on the above considerations, in the subsequent calculation of the profitability of the electricity energy market, it is assumed that the ratio of the peak load price to the valley price is controlled within 1.5 times. Take Pushihe PHES in Liaoning Province as an example. It is assumed that the PHES can benefit from not only the electricity energy market, but also the peak load shaving. It means that the revenue of this PHES is composed of two parts if it participates in the electricity market. In addition to the revenue of electricity energy brought by peak-valley price difference; it is also possible to obtain income from providing peak load shaving.

For the revenue obtained from the electricity energy market, the measured results (Table 2 and Table 3 show the parameters and corresponding values) show that in the ideal situation, when the PHES totally undertakes the peak peaking task of the Northeast Power Grid, the total revenue of the PHES still has a large gap with the current capacity fee (about 600 million Yuan). Under the assumption that the generating price is 1.5 times of the pumping price, the proportion of the revenue obtained from the energy market accounts for the capacity fee within $20 \%$.

For the revenue obtained from the peak load shaving, according to the latest rules of Northeast Ancillary Power Service Market listed in table 4, the revenue scale depends on the depth of peak regulation and the utilization hours. According to the comparison between the utilization hours of pumping water in the previous year and the national average, the pumping power amount higher than the national average shall be compensated after the fact in the current year. The specific compensation principles are as follows: Pumping hours is higher than the national average utilization hours 0 to $100 \%$ range, this part of the electricity will be compensated by $0.01 ¥ / \mathrm{kWh} ; 100$ to $200 \%$ range, $0.02 ¥ / \mathrm{kWh}$ to compensate; $200 \%$ of the above range, $0.03 ¥ / \mathrm{kWh}$ to compensate.

In 2016, the average pumping utilization hours of PHESs belong to the State Grid Xinyuan Company Ltd. was $2000 \mathrm{~h}$, assuming that the annual utilization hours of the Pushihe PHES in Northeast China Power Grid are 2 times of the national average, namely 4,000 hours (for very extreme cases). According to the price compensation principle shown in table 4, the extra 2000 hours will be compensated by $0.01 ¥ / \mathrm{kWh}$, so the proceeds will be $1200 \mathrm{MW} \times 2000 \mathrm{~h} \times 0.01 ¥ / \mathrm{kWh}=24$ million Yuan. To summarize, if Pushihe PHES participate in the electricity market, the revenue scale is only $0.736 \times 100+24=97.6$ million Yuan. However, the capacity fee will reach to 600 million Yuan annually, the revenue scale obtained in the competition market only accounts for $16.2 \%$. The results indicate that only participating in the market to obtain revenue cannot meet the demand for capacity fee recovery. 
Table 1. Comparison of average peak-valley pool purchase price in major electricity markets.

\begin{tabular}{cccc}
\hline Country & Average peak price & Averaege valley price & Peak-valley price ratio \\
\hline Australia & $41 \mathrm{AUD} / \mathrm{MWh}$ & $21 \mathrm{AUD} / \mathrm{MWh}$ & 1.95 \\
USA PJM & $40.97 \$ / \mathrm{MWh}$ & $28.11 \$ / \mathrm{MWh}$ & 1.46 \\
Zhejiang province & $0.5 ¥ / \mathrm{kWh}$ & $0.25 ¥ / \mathrm{kWh}$ & 2 \\
\hline
\end{tabular}

Table 2. Important arguments values for case study.

\begin{tabular}{cccccc}
\hline Parameters & $\begin{array}{c}\text { Capacity } \\
(\mathrm{MW})\end{array}$ & $\begin{array}{c}\text { Design power } \\
\text { generation hours } \\
(\mathrm{hr})\end{array}$ & $\begin{array}{c}\text { PHES efficiency } \\
(\%)\end{array}$ & $\begin{array}{c}\text { Generating } \\
\text { electricity price } \\
(¥ / \mathrm{kWh})\end{array}$ & $\begin{array}{c}\text { Pumping } \\
\text { electricity price } \\
(¥ / \mathrm{kWh})\end{array}$ \\
\hline Value & 1200 & 1500 & 75 & $0.3685^{\mathrm{a}}$ & $0.2457^{\mathrm{b}}$ \\
\hline
\end{tabular}

price of electricity. Therefore, the size of the entire

Table 3. PHES annual revenue profile (unit: MW, 100 million yuan).

\begin{tabular}{cc}
\hline Attributes & Value \\
\hline capacity (MW) & 1200 \\
Annual power generation revenue & 6.633 \\
Annual pumping cost & $5.897^{\mathrm{a}}$ \\
Electricity energy market revenue & 0.736 \\
\hline
\end{tabular}

Table 4. The price compensation principe for PHES.

\begin{tabular}{cc}
\hline Range & Subsidy Price \\
\hline $0 \sim 100 \%$ & $0.01 ¥ / \mathrm{kWh}$ \\
$100 \sim 200 \%$ & $0.02 ¥ / \mathrm{kWh}$ \\
Above $200 \%$ & $0.03 ¥ / \mathrm{kWh}$ \\
\hline
\end{tabular}

\subsection{Mature electricity market stage}

With reference to foreign experience, at the mature stage, the PHES can participate in the electric energy market and the ancillary energy markets such as AGC and reserve and other ancillary services markets. In the electric energy market, PHES draws water when the spot price is low and generates electricity when the spot price is at its peak. In this way, it gains profit from the "low buy and high sell" of electric energy considering the pumping loss. In the ancillary service market, the PHES can participate in AGC and compete in the reserve market to provide responsive service and gain market benefits. In addition, services such as black-start, emergency backup that are unsuitable for the short-term market can be procured by the system's security management authority and reimbursed for expenses through cost-based rates.

Taking the Pushihe PHES in northeastern China as an example, we calculate the profitability of the storage power station through the electric energy market and various ancillary service markets. According to international experience, the price of ancillary services is very low, which is generally only $1 / 50$ to $1 / 10$ of the

a. Liaoning Province unified coal-fired unit benchmark electricity price (tariff included). b. According to respectively $2 / 3$ of the on-grid electricity price, that is, the on-grid electricity price is 1.5 times of the pumping electricity price

c. The calculation of the electricity price of the Internet is 1.5 times as much as the price of the pumped electricity. ancillary service market is limited. In particular, at present, the National Energy Administration of China is promoting the reform of thermal power flexibility across the country and the flexibility of thermal power generation will be greatly increased in the future. Compared with conventional thermal power, PHES plants have no significant advantages in providing peak shaving, AGC, and routine back-up, and the benefits obtained are relatively low even if they participate in ancillary service market,. With reference to foreign experience, assuming that all types of ancillary services undertaken by the load bear the cost of $0.01 / \mathrm{kWh}$ Yuan and the scale of Liaoning's power generation is about 200 billion $\mathrm{kWh}$ in 2015 , the total ancillary service cost will be 2 billion Yuan. The installed capacity of Pushihe PHES is $1200 \mathrm{MW}$, and it accounts for nearly $3 \%$ of the total province installation capacity of 43,000 MW. Assuming the Pushihe PHES can receive ancillary service compensation proportional to its capacity share, it can only get 60 million Yuan. Considering the revenue from the electric energy market, the ratio between the revenue and the required electricity tariff is between $22 \%$ and $70 \%$, and there is still a large funding gap with the current capacity electricity tariff. The results are illustrated in table 5, and two other scenarios are also listed in the table for different peak-valley price ratios in order to model various electricity market environmental situations. It can be found that the peak-valley price ration has an obvious impact on the revenue of PHES.

It is noted that Case 2 and Case 3 here are difficult to achieve in practice. On the one hand, it is assumed that the calculation result is that PHES pumping and generating time $100 \%$ are used for peak shaving and valley filling. In actual operation, this is impossible to achieve the ideal situation; on the other hand, in order to reduce market risk and ensure the enthusiasm for investment in a power station, the mainly power plant investors hope that most of its proceeds in the early stage are guaranteed.

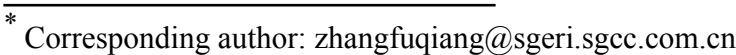


Table 5. Total revenue estimation when phes participated in the energy and ancillary services market (Unit: 100 Million Yuan).

\begin{tabular}{cccccc}
\hline Cases & $\begin{array}{c}\text { Peak-valley } \\
\text { price ratio }\end{array}$ & $\begin{array}{c}\text { Energy market } \\
\text { revenue }\end{array}$ & $\begin{array}{c}\text { Ancillary services } \\
\text { market revenue }\end{array}$ & Total revenue & $\begin{array}{c}\text { Ratio of total } \\
\text { revenue/capacity fees }\end{array}$ \\
\hline Case 1 & 1.5 & 0.736 & 0.6 & 1.336 & $22 \%$ \\
Case 2 & 2 & 2.211 & 0.6 & 2.811 & $47 \%$ \\
Case 3 & 3 & 3.685 & 0.6 & 4.285 & $70 \%$ \\
\hline
\end{tabular}

\section{Recommendations for future pricing mechanism}

According to the full-featured, unique advantages and universal service features of PHES, the following suggestions are made for the electricity pricing mechanism of PHES in combination with the current construction of power market in China.

(1) The pricing mechanism: According to the No. 1763 Document of the National Development and Reform Commission in 2014, the two-part tariff is still recommended to implement in current stage. Among them, the capacity-tariff mainly reflects the value of ancillary services such as capacity reserve, frequency regulation, phase modulation and black start provided by the PHES, which is set to make up the fixed costs and allowable profits of the PHES. The energy-tariff basically reflects the energy value provided by the PHES such as peak shaving, and its price is determined by the pumped storage power generation losses and other variable costs.

(2) Cost sharing mechanism: It is suggested the cost of PHES should be included in the allowable cost of power transmission and distribution, and be recovered together with the transmission and distribution tariff. Considering that the beneficiaries of PHES are not rather specific power plants, but the whole power systems, so it is not easy to distinguish accurately the beneficiaries. In order to ease the pressure of PHES cost recovery, the capacity-tariff and energy-tariff should be shared by all users in order to reflect the principle of equitable sharing. The cost of PHES can be taken along with the transmission and distribution tariff to be recovered. The allowable cost of the power grid is specified in the transmission and distribution tariff determination method. It includes the "ancillary service" fee (that is, the electricity capacity of the capacity) purchased by the grid enterprises from PHES. To ensure the normal operation, the PHES in operation and forthcoming operation should be accurately included in the transmission and distribution tariff approval cycle. In order to reflect the principle of "who benefits, who pay", we should clarify the scope and extent of the benefits of PHES so that we can extend the PHES tariffs to all beneficiary provinces in a region.

(3) Explore diverse funding sources. Three possible ways can be used to revenue the cost of PHES, and they are illustrated as follows. First, the PHES can stabilize the power output of renewable energy such as solar and wind power and consequently reduce the curtailment scale of renewable energy. The benefits produced by reducing the curtailment of solar and wind energy can cover part of the cost of PHES. For example, in the first half of 2017, the curtailment rate of wind power in China decreased by $7 \%$ and $4.5 \%$ respectively over the same period of previous year, and the corresponding economic benefits were about 15.4 billion Yuan. At present, ultrahigh voltage transmission technique is the most effective way to solve the high-level energy curtailment. Besides, PHES plays an important role in the power supply side, grid side and load side to improve the efficiency of renewable energy utilization. Therefore, the benefits from wind power and photovoltaic power generation can pay part of the service fee to PHES. Second, it is suggested that economic benefits from increasing utilization hours of nuclear power plants or not participating in peak regulation should be deducted, and an appropriate proportion should be deducted from the nuclear power price to compensate the PHES operate costs. Third, explore the establishment of a nationwide dispatch service fund to solve the problem of compensating for operating expenses before the newlystarted PHES are not diverted.

(4) Multi-channel electricity pricing mechanism adopting "fixed purchasing+market bidding" in the market environment should be recommended. On the one hand, considering that the PHES affects the emergency reserve function when participating in the market competition, the main function such as black start means that it cannot compete freely with other power sources in the market. On the other hand, some functions provided by PHES such as black start-up and reactive power support have no corresponding market, which means the market cannot fully reflect the benefit of the PHES in power systems.

In view of the special properties and attributes of PHES, it is suggested to adopt multiple channels of "fixed purchasing+market bidding" to solve the costrevenue of PHES in electricity market environment. First, about $80 \%$ of the PHES costs are secured through longterm fixed contracts with the electricity trading agencies, and the electricity trading agencies will charge the fixed service ancillary services with the power to all users. It is a compensation for ancillary services that are not suitable for market-oriented such as emergency reserve, black-start and reactive power support for PHES. For this part, it is recommended these functions should adopt the mode of "fixed purchasing" to ensure a reasonable and reliable revenue.

Second, for the remaining about $20 \%$ of the cost of PHESs, they can participate in the energy market and ancillary service market in accordance with market rules, obtain revenue through market competition, and compensate for the shortage of fixed contracts.

\section{Conclusion}

This paper first stated the challenges the PHES faced in the progress of transmission and distribution tariff 
reform. It is necessary to propose a reasonable pricing mechanism for the healthy development of PHES, we review and summarize international PHES development experience and conduct a comparative analysis of revenue mechanism in different markets. The main challenges and barriers to PHES development in China are also analyzed. In order to measure the impact of electricity tariff reform on the PHES, the revenue scale is estimated respectively in China's different marketoriented reform phase, namely the transitional phase and the mature power market stage. It indicates that the revenue gained by participating in the electricity market competition is not enough for PHES, and the majority of the cost shoul be covered by capacity-tariff. Based on the research, four recommendations are proposed for the policy makers. The two-part tariff scheme is proposed in China considering the special characteristics of PHES, of which the capacity-tariff is used to cover the about $80 \%$ of the cost and the energy-tariff is designed to compensate the variable cost of PHES.

\section{Acknowledgements}

This work is supported by Science and Technology Project of State Grid Corporation of China (B3670116A005): Study on the evaluation model and application of peak shaving technology, economy and policy for power system wiht large-scale renewable energy integration.

\section{References}

1. Development and reform commission of China (NDRC), Notice on improving price formation mechanism of pumped hydro energy storage (No.1763)

http://jgs.ndrc.gov.cn/zcfg/201408/t20140812_6220 $\underline{12 . h t m l}$ (2014)

2. Development and reform commission of China (NDRC), measures for electricity tariff determination for transmission and distribution of provincial power grid (Trial) http://www.ndrc.gov.cn/gzdt/201701/t20170104_83 4330.html (2014)

3. C. Chusheng, Hydropower Pumped Storage, 3, 3 (2017)

4. L. Zhiwei, L. Qinghua, H. Shuangcheng, Water Power, 43, 5 (2017)

5. C. Shangfa, Electr. Power Syst. Equip., 4 (2008)

6. S. Zhongming, J. Wuhan Polytech. Coll., 4 (2014)

7. Z. Quanren, Hunan Electr. Power, 1 (1998)

8. L. Shasha, L. Yun, L. Guozhong, Sino-Global Energy, 18 (2013)

9. E. Barbour, I. A.G. Wilson, J. Radcliffe, Renew. Sustain. Energy Rev., 61 (2016)

10. China Smart Grid : Pumping stations: the reasonable price mechanism has not been how to make the best use, http://www.chinasmartgrid.com.cn/news/20150807/ 607957-4.shtml. (2014)

11. M. Hamada, V. D. H. John, Social Science Electronic Publishing (2011)

12. M. Analytics, State of the Market Report for PJM (2016)

13. Z. Fuqiang, Z. Hao, Autom. Electr. Power Syst. 30, 4 (2006) 